\title{
Spacetime noncommutativity and ultra-high energy cosmic ray experiments
}

\author{
Raul Horvat, ${ }^{1}$ Dalibor Kekez, ${ }^{1}$ and Josip Trampetić ${ }^{1}$ \\ ${ }^{1}$ Physics Division, Rudjer Bošković Institute, Zagreb, Croatia
}

\begin{abstract}
If new physics were capable to push the neutrino-nucleon inelastic cross section three orders of magnitude beyond the standard-model (SM) prediction, then ultra-high energy (UHE) neutrinos would have already been observed at neutrino observatories. We use such a constraint to reveal information on the scale of noncommutativity (NC) $\Lambda_{\mathrm{NC}}$ in noncommutative gauge field theories (NCGFT) where neutrinos possess a tree-level coupling to photons in a generation-independent manner. In the energy range of interest $\left(10^{10}\right.$ to $\left.10^{11} \mathrm{GeV}\right)$ the $\theta$-expansion $\left(|\theta| \sim 1 / \Lambda_{\mathrm{NC}}^{2}\right)$ and therefore the perturbative expansion in terms of $\Lambda_{\mathrm{NC}}$ retains no longer its meaningful character, forcing us to resort to those $\mathrm{NC}$ field-theoretical frameworks involving the full $\theta$-resummation. Our numerical analysis of the contribution to the process coming from the photon exchange, pins impeccably down a lower bound on $\Lambda_{\mathrm{NC}}$ to be as high as around up to 900 (450) $\mathrm{TeV}$, depending on the estimates for the cosmogenic neutrino flux. If, on the other hand, one considers a surprising recent result occurred in Pierre Auger Observatory (PAO) data, that UHE cosmic rays are mainly composed of highly-ionized Fe nuclei, then our bounds get weaker, due to the diminished cosmic neutrino flux. Nevertheless, we show that even for the very high fraction of heavy nuclei in primary UHE cosmic rays, our method may still yield remarkable bounds on $\Lambda_{\mathrm{NC}}$, typically always above $200 \mathrm{TeV}$. Albeit, in this case one encounters a maximal value for the Fe fraction from which any useful information on $\Lambda_{\mathrm{NC}}$ can be drawn, delimiting thus the applicability of our method.
\end{abstract}

PACS numbers: 11.10.Nx; 13.15.+g; 13.60.Hb; 98.70.Sa

The observation of ultra-high energy (UHE) neutrinos from extraterrestrial sources would open a new window to look to the cosmos, as such neutrinos may easily escape very dense material backgrounds around local astrophysical objects, giving thereby information on regions that are otherwise hidden to any other means of exploration. In addition, neutrinos are not deflected on their way to the earth by various magnetic fields, pointing thus back to the direction of distant UHE cosmic-ray source candidates. This could also help resolving the underlying acceleration in astrophysical sources.

Since the GZK-structure in the energy spectrum of UHE cosmic rays at $\sim 4 \times 10^{19} \mathrm{eV}$ has been observed recently with high statistical accuracy [1], the flux of the so-called cosmogenic neutrinos, arising from photopion production on the cosmic microwave background $p \gamma_{C M B} \rightarrow \Delta^{*} \rightarrow N \pi$ and subsequent pion decay, is now guaranteed to exist. Although estimates for the cosmogenic neutrino flux are very model-dependent, primarily due to our insufficient knowledge of the nature and the origin of UHE cosmic rays, possible ranges for the size of the flux of cosmogenic neutrinos can be obtained from separate analysis of the data from various largescale observatories 2, 3]. With this knowledge of cosmogenic neutrinos and the increased exposure of large-scale detectors, a tight model-independent constraint on the neutrino-nucleon cross section can be placed [4]. A similar analysis but with a different approach to the problem was carried out in [5].

We note that the uncertainty in the flux of cosmogenic neutrinos may be further aggravated in view of apparently contradictory recent results regarding the chemical composition of UHE cosmic rays. While the Pierre Auger
Observatory (PAO) data indicate that UHE cosmic rays may be highly-ionized Fe nuclei [6], the results from the High Resolution Fly's Eye Collaboration (HiRes) are consistent with the proton dominance in the UHE region [7]. In the former case the flux of cosmogenic neutrinos would possibly be much smaller than that in the latter case [8]. Further confusion was brought in by the fact that both experiments are intrinsically inconsistent regarding the arrival directions of UHE cosmic rays [9]. Note also that the most recent evidence of anisotropy of UHE cosmic-ray arrival directions at the $99 \%$ confidence level [10] by the PAO would question further its interpretation that primary UHE cosmic rays are likely to be dominated by heavy nuclei. On top of that, there is another inconsistency besetting the PAO data: the average of the depth in the atmosphere at which the shower contains the largest number of high energy particles implies an Fe fraction $<60 \%$, whereas the analysis of its standard deviation implies for the same fraction to be $>90 \%$. Although previously most observations and arguments seemed to favor protons as a dominant component at the highest energies, and although some speculations exist that inconsistencies in the PAO data rather point to some new physics than to a heavy component in the UHE cosmic rays [11], this issue still remains an open question 12].

In this paper, we employ the aforementioned upper bound [4] on the cross section to constrain the scale of noncommutativity entering NCGFT in which neutrinos couple directly to photons. We use upper bounds derived by non-observation of UHE neutrino-induced events in RICE Collaboration search results [13] and exploiting the predictions for the cosmogenic neutrino flux from 
[2, 3]. Also, to realize the second possibility as indicated by the PAO data, we derive our bounds by using the same predictions for the cosmogenic neutrino flux, but now diminished by the fraction of FE nuclei in the UHE portion of cosmic ray spectrum, which we treat here as a free parameter.

NCGFT that we consider is based on Moyal-Weyl star $(\star)$-product [41] and it belongs to a class of models that expand the $\mathrm{NC}$ action in $\theta$ before quantization [14, 15]. In these models Seiberg-Witten (SW) maps [16] are necessary to express the noncommutative fields $\widehat{\psi}$ and $\widehat{A}_{\mu}$, that appear in the action and transform under NC gauge transformations, in terms of their asymptotic commutative counterparts $\psi$ and $A_{\mu}$. The algebra generated here is the enveloping algebra. Computation of any (physical) quantities, obtained in this approach, are given as a power series in the $\mathrm{NC}$ parameter $\theta$, thus showing no UV/IR mixing on the quantum level.

Admittedly, in other approaches, with $\theta$-unexpanded, i.e., models based on $\star$-products only [17], the celebrated quantum-gravity effect shows up: the UV/IR mixing 18 20]. The same would happen in our approach.

Coupling of matter fields to Abelian gauge bosons is a $\mathrm{NC}$ analogue of the usual minimal coupling scheme. It will imply only a vector-like NC coupling between photons and neutrinos in the $\mathrm{U}(1)_{\star}$ gauge-invariant way, thus endowing also otherwise sterile right-handed $(\mathrm{RH})$ neutrino components a new interaction.

It is possible to extend the model [15] to the NC electroweak model based on the other gauge groups, like NC version of the standard model (NCSM), etc. [21, 22]. That NCSM 21] at $\theta$-order does break Lorentz symmetry, appears to be anomaly free [23] and it does have remarkable well behaved one-loop quantum corrections 24], as well as some other models do [25 27].

Signatures of noncommutativity and/or the bounds on the NC scale come from neutrino astrophysics [15, 28, 29] and cosmology [30, 31]; from high energy particle physics 32 34], producing a scale of noncommutativity of order few TeV's. Typical low energy nonaccelerator experiments are the Lamb-shift [35], and clock-comparison experiments [36]. The bound from $Z \rightarrow \gamma \gamma$ decay on $\Lambda_{\mathrm{NC}}$, of order a few TeV's [33], is the most robust due to the finite one-loop quantum corrections in the gauge sector of the nmNCSM 24]. Also, the interesting upper bound on the scale of NC, of order of one tenth of the Planck mass, can be obtained from the black-hole physics in the NC spacetime [37].

Neutrinos do not carry an electromagnetic charge and hence do not couple directly to photons. However, in the presence of spacetime $\mathrm{NC}$, it is possible to couple neutral particles to photons via a $\star$-covariant derivative $\widehat{D}_{\mu} \widehat{\psi}$. Thus, the action for neutrinos that couples to an Abelian gauge boson in the $\mathrm{NC}$ background, invariant under the NC gauge transformations, is

$$
\begin{aligned}
S_{\mathrm{NC}} & =\int d^{4} x\left(\overline{\widehat{\psi}} \star i \gamma^{\mu} \widehat{D}_{\mu} \widehat{\psi}-m \overline{\widehat{\psi}} \star \widehat{\psi}\right) \\
\widehat{D}_{\mu} \widehat{\psi} & =\partial_{\mu} \widehat{\psi}-i \kappa e\left[\widehat{A}_{\mu}, \widehat{\psi}\right] \\
\widehat{A}_{\mu} & =A_{\mu}+e \theta^{\nu \rho} A_{\rho}\left[\partial_{\nu} A_{\mu}-\frac{1}{2} \partial_{\mu} A_{\nu}\right]+\mathcal{O}\left(\theta^{2}\right), \\
\widehat{\psi} & =\psi+e \theta^{\nu \rho} A_{\rho} \partial_{\nu} \psi+\mathcal{O}\left(\theta^{2}\right),
\end{aligned}
$$

where (3) and (44) are, up to the first order in $\theta$, ordinary SW maps for the Abelian NC gauge potential and "chiral" SW map for the NC "chiral" neutrino field, respectively [42]. Here, we fix the NC scale by defining $\theta^{\mu \nu} \equiv c^{\mu \nu} / \Lambda_{\mathrm{NC}}^{2}$, such that the matrix elements of $c$ are of order one.

Performing the SW and $\star$-product expansions up to the first order in $\theta$ one obtains the following electromagnetically gauge-invariant $\mathrm{NC}$ action for photons and neutrinos in terms of commutative fields and parameters [15]:

$$
S_{\mathrm{NC}}=-\frac{e}{2} \int d^{4} x \bar{\psi} A_{\mu \nu}\left(i \theta^{\mu \nu \rho} \partial_{\rho}-\theta^{\mu \nu} m\right) \psi,
$$

with $\theta^{\mu \nu \rho}=\theta^{\mu \nu} \gamma^{\rho}+\theta^{\nu \rho} \gamma^{\mu}+\theta^{\rho \mu} \gamma^{\nu} ; A_{\mu \nu}=\partial_{\mu} A_{\nu}-\partial_{\nu} A_{\mu}$. From (5) we extract the Feynman rule for massless left/right $(\mathrm{L} / \mathrm{R})$-handed neutrinos (the same for each generation and $\left.k=k^{\prime}+q\right)$ [15]:

$$
\Gamma_{\left(\begin{array}{l}
\mathrm{L} \\
\mathrm{R}
\end{array}\right)}^{\mu}(\bar{\nu} \nu \gamma)=\frac{i}{2} e\left(1 \pm \gamma_{5}\right) \theta^{\mu \nu \rho} k_{\nu} q_{\rho} .
$$

The double differential deep inelastic scattering cross section $d^{2} \sigma_{\mathrm{NC}} / d x d y$ for the process $\nu N \rightarrow \nu+$ anything via the $\bar{\nu} \nu \gamma$ vertex as given by ([6), can be calculated with the help of the structure functions,

$$
\begin{aligned}
& \frac{d^{2} \sigma_{\mathrm{NC}}}{d x d y}=\mathcal{I} \frac{d^{2} \sigma_{\mathrm{comm}}}{d x d y}, \\
& \mathcal{I}=\frac{1}{2 \pi} \int_{0}^{2 \pi} d \varphi\left(\frac{k c k^{\prime}}{2 \Lambda_{\mathrm{NC}}^{2}}\right)^{2} \\
& \approx\left(\left(c_{01}-c_{13}\right)^{2}+\left(c_{02}-c_{23}\right)^{2}\right) \frac{E_{\nu}^{3} M_{N}}{4 \Lambda_{\mathrm{NC}}^{4}} x y(1-y), \\
& \frac{d^{2} \sigma_{\mathrm{comm}}}{d x d y}=\frac{2 \pi \alpha^{2}}{E_{\nu} M_{N}(x y)^{2}} \\
& \times\left[(1-y) F_{2}^{\gamma}+y^{2} x F_{1}^{\gamma}+y(1-y / 2) x F_{3}^{\gamma}\right] .
\end{aligned}
$$

Here the Bjorken variable $x$ is given in terms of the inelasticity $y$, the total energy of the system $s$ and the invariant momentum transfer $-Q^{2}$ as $x=Q^{2} /(y s)$. The azimuth angle $\varphi$ in (8) is defined with respect to the direction of the incident neutrino. The quantity $\sigma_{\text {comm }}$ refers to the cross section a neutrino would have as if it were a Weyl particle with a charge $e$. The structure function $F_{i}$ 's for the isoscalar nucleon are given by

$$
2 x F_{1}^{\gamma}\left(x, Q^{2}\right)=F_{2}^{\gamma}\left(x, Q^{2}\right),
$$




$$
\begin{aligned}
\frac{1}{x} F_{2}^{\gamma}\left(x, Q^{2}\right) & =\frac{1}{2}\left(Q_{u}^{2}+Q_{d}^{2}\right)\left[u\left(x, Q^{2}\right)+\bar{u}\left(x, Q^{2}\right)\right. \\
& \left.+d\left(x, Q^{2}\right)+\bar{d}\left(x, Q^{2}\right)\right] \\
& +2 Q_{c}^{2} c\left(x, Q^{2}\right)+2 Q_{s}^{2} s\left(x, Q^{2}\right) \\
& +2 Q_{b}^{2} b\left(x, Q^{2}\right)+2 Q_{t}^{2} t\left(x, Q^{2}\right) \\
F_{3}^{\gamma}\left(x, Q^{2}\right) & =0
\end{aligned}
$$

and in our analysis we use the parton distribution functions from the audibly available package CTEQ4 [38]. The kinematical region of interest here is that of high $Q^{2}$ and very small $x$ values $x \approx 1.7 \times 10^{-7} /\left(E_{\nu} / 10^{11} \mathrm{GeV}\right)$, because of the rapid increase of the parton densities towards the small $x$ 's. Different extrapolation approaches can result in uncertainties as large as a factor of two at $E_{\nu}=10^{12} \mathrm{GeV}[39$.

Employing the upper bound on the $\nu N$ cross section derived from the RICE Collaboration search results 13 at $E_{\nu}=10^{11} \mathrm{GeV}\left(4 \times 10^{-3} \mathrm{mb}\right.$ for the FKRT neutrino flux [2])), one can infer from (66]12) [also employing $\left.\left(c_{01}-c_{13}\right)^{2}+\left(c_{02}-c_{23}\right)^{2}=1\right]$ on the scale of noncommutativity $\Lambda_{\mathrm{NC}}$ to be greater than $455 \mathrm{TeV}$, a really strong bound. One should however be careful and suspect this result as it has been obtained from the conjecture that the $\theta$-expansion stays well-defined in the kinematical region of interest, and the more reliable limits on $\Lambda_{\mathrm{NC}}$ are expected to be placed precisely by examining low-energy processes [29]. Although a heuristic criterion for the validity of the perturbative $\theta$-expansion, $\sqrt{s} / \Lambda_{\mathrm{NC}} \lesssim 1$, with $s=2 E_{\nu} M_{N}$, would underpin our result on $\Lambda_{\mathrm{NC}}$, a more thorough inspection on the kinematics of the process does reveal a more stronger energy dependence $E_{\nu}^{1 / 2} s^{1 / 4} / \Lambda_{\mathrm{NC}} \lesssim 1$. In spite of an additional phase-space suppression for small $x$ 's in the $\theta^{2}$-contribution [34] of the cross section relative to the $\theta$-contribution, we find an unacceptably large ratio $\sigma\left(\theta^{2}\right) / \sigma(\theta) \simeq 10^{4}$, at $\Lambda_{\mathrm{NC}}=455$ $\mathrm{TeV}$. Hence, the bound on $\Lambda_{\mathrm{NC}}$ obtained this way is incorrect, and our last resort is to modify the model adequately to include somehow the full- $\theta$ resummation, thereby allowing us to compute nonperturbatively in $\theta$.

The simplest possible modeling, which we propose here is: (a) Replacement of the NC fields with the commutative ones, $\widehat{\psi} \rightarrow \psi$ and $\widehat{A}_{\mu} \rightarrow A_{\mu}$; (b) Expansion of the «-product up to all orders, and resummation to the $\theta$ exact final results. Following (a) and (b), the NC action instead of (1) can be written now as

$$
S_{\mathrm{NC}}(\theta)=-i e \int d^{4} x \bar{\psi} \gamma^{\mu}\left(A_{\mu} \star \psi-\psi \star A_{\mu}\right) .
$$

At this point the above action corresponds to the neutrino-photon interaction proposed in Ref. 17]. Next we expand the $\star$-product to all orders in $\theta$, and take derivatives on $\psi(x) \sim e^{i k x} \tilde{\psi}(k)$ and $A_{\mu}(x) \sim e^{i q x} \tilde{A}_{\mu}(q)$. After getting the momentum dependence order by order we resume the obtained series into the nontrivial exponential phase factors $\exp \left( \pm i \frac{q \theta k}{2}\right)$, and extract a generation-independent Feynman rule for massless L/R neutrinos

$$
\Gamma_{\left(\begin{array}{l}
\mathrm{L} \\
\mathrm{R}
\end{array}\right)}^{\mu}(\bar{\nu} \nu \gamma)=i e\left(1 \pm \gamma_{5}\right) \gamma^{\mu} \sin \left(\frac{q \theta k}{2}\right) .
$$

By comparison of our Feynman rule (14) with those from [18, 20], it is clear that our model would also produce the UV/IR mixing in computing quantum corrections, thus, our model is not perturbatively renormalizable and it is not clear how to interprete the quantum corrections and to relate them to the observations [40]. In return, we obtain the $\theta$ well-behaved deep inelastic cross section at ultra-high energies.

With the aid of the full vertex (14), the neutrino cross section still retains the form (77), but with the relevant quantity now defined as

$$
\begin{aligned}
\mathcal{I} & =\frac{1}{2 \pi} \int_{0}^{2 \pi} d \varphi 4 \sin ^{2}\left(\frac{k c k^{\prime}}{2 \Lambda_{\mathrm{NC}}^{2}}\right) \\
& =2\left(1-\cos (A) J_{0}(B)\right),
\end{aligned}
$$

where $J_{0}$ is the Bessel function of the first kind of order zero, and

$$
\begin{aligned}
A & =\frac{E_{\nu} E_{\nu}^{\prime}}{\Lambda_{\mathrm{NC}}^{2}} c_{03}(\cos \vartheta-1) \\
B & =\frac{E_{\nu} E_{\nu}^{\prime}}{\Lambda_{\mathrm{NC}}^{2}} \sin \vartheta \\
& \times \operatorname{sign}\left(c_{01}-c_{03}\right) \sqrt{\left(c_{01}-c_{13}\right)^{2}+\left(c_{02}-c_{23}\right)^{2}} .
\end{aligned}
$$

The behavior of the cross section with the scale of noncommutativity at fixed $E_{\nu}=10^{10} \mathrm{GeV}$ and $E_{\nu}=10^{11}$ $\mathrm{GeV}$, together with the upper bounds depending on the actual size of the cosmogenic neutrino flux (FKRT [2] and PJ [3]) as well as the total SM cross sections at these energies, are depicted in our Figure 1. In order to maximize the effect of noncommutativity, in our numerical calculations we choose $c_{01}-c_{13}=c_{02}-c_{23}=c_{03}=1$.

A few comments are in order regarding qualitative behavior of the cross section. The common attribute for both energies is the existence of a plateau at small $\Lambda_{\mathrm{NC}}$ 's, where the cross section tends to a constant value. Also, the oscillatory term in (15) enters the regime of rapid oscillations for lower $\Lambda_{\mathrm{NC}}$ 's for both energies. This behavior can be read off from (15) by noting that the argument of the cosine function grows as $\Lambda_{\mathrm{NC}}$ is decreasing (for fixed energies), whilst the amplitude of oscillations as given by $J_{0}$ tends to zero asymptotically in the same limit. The cross section for the upper energy does clearly surpass the measured value below around 900 (450) TeV for the FKRT (PJ) flux, bringing us a valuable information on the NC scale. As far as the lower energy is concerned, the upper bounds assigned to both fluxes are crossed, at energies around $350(500) \mathrm{TeV}$, respectively. Thus, the conservative bound for the energy $E_{\nu}=10^{10} \mathrm{GeV}$ is around $350 \mathrm{TeV}$. 
If future data confirm that UHE cosmic rays are composed mainly of Fe nuclei, as indicated, for the time being, by the PAO data, then still valuable information on $\Lambda_{\mathrm{NC}}$ can be obtained with our method, as seen in Fig. 2. Here we see the intersections of our curves with the RICE results (cf. Fig.1) as a function of the fraction $\alpha$ of Fe nuclei in the UHE cosmic rays. We see that in all but one cases our method is capable to extract information on $\Lambda_{\mathrm{NC}}$ even for Fe fractions as large as $\gtrsim 90 \%$, giving always a conservative bound $\gtrsim 200 \mathrm{TeV}$. The terminal point on each curve gives the highest fraction of $\mathrm{Fe}$ beyond which our method becomes unserviceable to draw any information on the NC scale. Thus, when more data on $\mathrm{Fe}$ fraction become available, one can easily pinpoint the lower bound on $\Lambda_{\mathrm{NC}}$ with the aid of Fig. 2 .

To summarize, we have used the upper bound on the neutrino-nucleon inelastic cross section, derived by nonobservation of UHE neutrino-induced events in extensive airshower arrays, to deduce information on the scale of noncommutativity in NC gauge field theories. The fact that we deal here with a process whose center-of-mass energy is three orders of magnitude higher than that being achieved with terrestrial accelerators, has also implications for the theory itself. Namely, the dimensionless parameter involving the scale of noncommutativity and entering the perturbative expansion shows a strong incident-energy dependence, such that perturbative expansion at such UHE energies is no longer meaningful. After having treated our model nonperturbatively in $\theta$, we used such a model to derive a very strong bound on the scale of noncommutativity, at around 900 (450) TeV, depending on the model for the total cosmogenic neutrino flux. Since the prediction for the NC neutrino-photon vertex in a perturbative setting is quite robust amongst different models, we believe that our $\theta$-resummed interaction is also a generic one. For those inclined to accept the PAO interpretation, we have shown that even for the large fraction of Fe nuclei in the UHE cosmic rays ( $\gtrsim 90 \%$ ) one may obtain remarkable bounds on the scale of noncommutativity $\left(\Lambda_{\mathrm{NC}} \gtrsim 200 \mathrm{TeV}\right)$. Finally, one should keep in mind that the limits derived here are conservative ones, meaning that any influence of Fe nuclei as well as NC physics on the cosmogenic neutrino flux (and consequently on the experimental upper limit on the cross section) has not been treated.

\section{Acknowledgment}

The work of R.H., D.K and J.T. are supported by the Croatian Ministry of Science, Education and Sports under Contract Nos. 0098-0982930-2872 and 0098-09829302900, respectively. We would like to thank P. Schupp and J. You for useful discussions, and W. Hollik at MPI for the hospitality. The work of J.T. is in part sup-

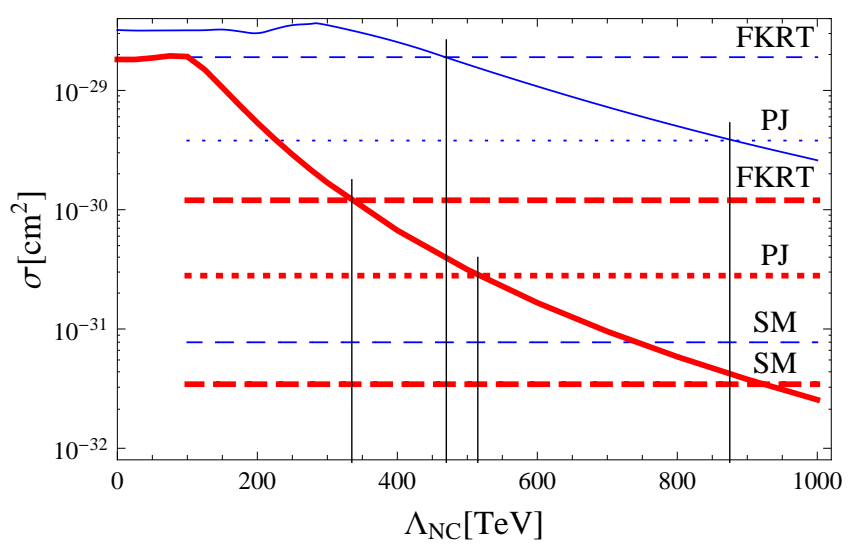

FIG. 1: $\nu N \rightarrow \nu+$ anything cross sections vs. $\Lambda_{\mathrm{NC}}$ for $E_{\nu}=10^{10} \mathrm{GeV}$ (red lines) and $E_{\nu}=10^{11} \mathrm{GeV}$ (blue lines). FKRT and PJ lines are the upper bounds on the neutrinonucleon inelastic cross section, denoting different estimates for the cosmogenic neutrino flux (see the text). SM denotes the SM total (charged current plus neutral current) neutrinonucleon inelastic cross section. The vertical lines denote the intersections of our curves with the RICE results.

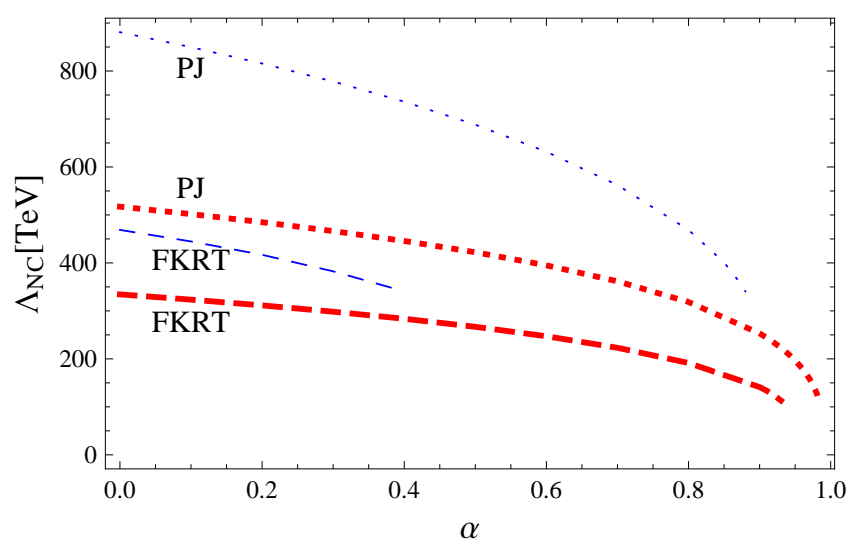

FIG. 2: The intersections of our curves with the RICE results (cf. Fig.1) as a function of the fraction of Fe nuclei in the UHE cosmic rays. The terminal point on each curve represents the highest fraction of Fe nuclei above which no useful information on $\Lambda_{\mathrm{NC}}$ can be inferred with our method.

ported by the HEPTOOLS under contract MRTN-CT2006-035505.

[1] J. Abraham et al. [Pierre Auger Collaboration], Astropart. Phys. 29, 243 (2008).

[2] Z. Fodor et al., JCAP 0311, 015 (2003).

[3] R. J. Protheroe and P. A. Johnson, Astropart. Phys. 4, 253 (1996).

[4] L. A. Anchordoqui et al., JCAP 0506, 013 (2005).

[5] V. Barger, P. Huber and D. Marfatia, Phys. Lett. B 642, 
333 (2006).

[6] J. Abraham et al. [Pierre Auger Observatory Collaboration], Phys. Rev. Lett. 104, 091101 (2010).

[7] R. U. Abbasi et al. [HiRes Collaboration], Phys. Rev. Lett. 104, 161101 (2010).

[8] M. Lemoine, Phys. Rev. D 71, 083007 (2005); K. Kotera and M. Lemoine, Phys. Rev. D 77, 123003 (2008).

[9] R. U. Abbasi et al., Astrophys. J. Lett. 713, L64 (2010).

[10] P. Abreu et al. [The Pierre Auger Collaboration], arXiv:1009.1855 [astro-ph.HE]; Accepted for publication in Astroparticle Physics on 31 August 2010.

[11] G. Wilk and Z. Wlodarczyk, arXiv:1006.1781 astro-ph].

[12] B. Schwarzschild, Phys. Today 63N5, 15 (2010).

[13] I. Kravchenko et al., Astropart. Phys. 20, 195 (2003).

[14] J. Madore et al. Eur. Phys. J. C 16, 161 (2000).

[15] P. Schupp et al., Eur. Phys. J. C 36, 405 (2004).

[16] N. Seiberg and E. Witten, JHEP 9909, 032 (1999).

[17] M. Chaichian et al., Eur. Phys. J. C 29, 413 (2003).

[18] C. P. Martin and D. Sanchez-Ruiz, Phys. Rev. Lett. 83, 476 (1999); A. Matusis, L. Susskind, and N. Toumbas, JHEP 0012, 002 (2000); L. Alvarez-Gaume and M. A. Vazquez-Mozo, Nucl. Phys. B 668, 293 (2003).

[19] S. A. Abel et al., JHEP 0609, 074 (2006).

[20] P. Schupp and J. You, JHEP 0808, 107 (2008).

[21] X. Calmet et al., Eur. Phys. J. C 23, 363 (2002); B. Melic et al., Eur. Phys. J. C 42, 483 (2005); ibid. 499; W. Behr et al., Eur. Phys. J. C 29, 441 (2003); G. Duplancic, P. Schupp, J. Trampetic, Eur. Phys. J. C 32, 141 (2003).

[22] P. Aschieri et al. Nucl. Phys. B 651, 45 (2003).

[23] C. P. Martin, Nucl. Phys. B 652 (2003) 72; F. Brandt, C. P. Martin, and F. R. Ruiz, JHEP 0307, 068 (2003).

[24] M. Buric, V. Radovanovic, and J. Trampetic, JHEP 0703, 030 (2007).

[25] A. Bichl et al., JHEP 0106, 013 (2001).

[26] D. Latas, V. Radovanovic, and J. Trampetic, Phys. Rev. D 76, 085006 (2007); C. P. Martin and C. Tamarit, Phys. Lett. B 658, 170 (2008); M. Buric et al., Phys. Rev. D 77, 045031 (2008); M. Buric et al., arXiv:1009.4603 [hep-th], to be published in Phys. Rev. D.

[27] M. M. Ettefaghi and M. Haghighat, Phys. Rev. D 77, 056009 (2008).

[28] P. Minkowski, P. Schupp, and J. Trampetic, Eur. Phys.
J. C 37, 123 (2004).

[29] M. Haghighat, Phys. Rev. D 79, 025011 (2009).

[30] E. Akofor et al. Phys. Rev. D 79, 063004 (2009).

[31] R. Horvat, J. Trampetic, Phys. Rev. D 79, 087701 (2009).

[32] B. Melic, K. Passek-Kumericki, and J. Trampetic, Phys. Rev. D 72, 054004 (2005); ibid 057502; C. Tamarit and J. Trampetic, Phys. Rev. D 79, 025020 (2009).

[33] M. Buric et al., Phys. Rev. D 75, 097701 (2007);

[34] A. Alboteanu, T. Ohl, and R. Ruckl, Phys. Rev. D 76, 105018 (2007).

[35] M. Chaichian, M. M. Sheikh-Jabbari, and A. Tureanu, Phys. Rev. Lett. 86, 2716 (2001); ibid. Eur. Phys. J. C 36, 251 (2004).

[36] S. M. Carroll et al., Phys. Rev. Lett. 87, 141601 (2001).

[37] P. Nicolini, A. Smailagic and E. Spallucci, Phys. Lett. B 632, 547 (2006); W. Kim and D. Lee, arXiv:1005.0459.

[38] H. L. Lai et al., Phys. Rev. D 55, 1280 (1997).

[39] K. Kutak and J. Kwiecinski, Eur.P.J. C 29, 521 (2003).

[40] R. Horvat and J. Trampetic, JHEP 1101, 112 (2011) arXiv:1009.2933 [hep-ph]].

[41] The Moyal-Weyl star( $(\star)$-product of some fields $f, g$, $(f \star g)(x)=f(x) e^{\frac{i}{2} \overleftarrow{\partial_{\mu}} \theta^{\mu \nu} \overrightarrow{\partial_{\nu}}} g(x)$, is a non-local bilinear expression in the fields and their derivatives, and it is associative but, in general, not commutative. It takes the form of a series in a Poisson antisymmetric tensor $\theta^{\mu \nu}$ defined via $\star$-commutator, $\left[x^{\mu}, x^{\nu}\right]_{\star}=x^{\mu} \star x^{\nu}-x^{\nu} \star x^{\mu}=$ $i \theta^{\mu \nu}$, which implements the spacetime NC. Also, the important property is $\int d^{4} x f \star g \star h=\int d^{4} x f \cdot(g \star h)$, for $\theta=$ constant.

[42] Note that a "chiral" Seiberg-Witten map is compatible with grand unified models where fermion multiplets are chiral [22]. In eqs (12) with chiral fermion fields, one may think of the NC neutrino field $\widehat{\psi}$ as having left charge $+\kappa e$, right charge $-\kappa e$ and total charge zero. Coupling $\kappa e$ corresponds to a multiple (or fraction) $\kappa$ of the electric charge $e$. From the perspective of non-Abelian gauge theory, one could also say that the neutrino field is charged in $\mathrm{NC}$ analogue of the adjoint representation with the matrix multiplication replaced by the $\star$-product. For this model where only the neutrino has dual L/R charges, $\kappa=1$ is required by the gauge invariance of the action. 